\title{
Complicações agudas da diabetes mellitus
}

\author{
ROSA GALLEGO,* JORGE CALDEIRA**
}

\section{RESUMO}

Introdução: As complicações agudas da diabetes (hipoglicemia, cetoacidose diabética, sindrome hiperglicémico hiperosmolar e lactoacidose) são causa de recorrência à urgência e internamento e estão associadas, na maioria dos casos, a intercorrências médicas e/ou falhas terapêuticas. São preveníveis pela educação terapêutica continuada da pessoa com diabetes e seus cuidadores, mas mantêm-se um problema actual, sobretudo nas crises de hiperglicemia com vómitos e desidratação, pela sua elevada incidência e mortalidade. 0 Médico de Família deve estar preparado para efectuar o diagnóstico e o tratamento atempados e contactar, o mais breve possivel, para eventual referenciação, a equipa dos cuidados secundários, assim que suspeitar de qualquer uma das situações.

Objectivos: Rever a patofisiologia, prevenção e tratamento das complicações agudas da diabetes mellitus, de forma a fornecer uma base para a construção de orientações técnicas a utilizar na prática da Medicina Geral e Familiar.

Métodos: Foi realizada uma pesquisa em bases de dados electrónicas ("Pubmed, Cochrane database of systematic reviews" e "Medline"), complementada com pesquisa manual em livros de texto. Os limites temporal (últimos cinco anos) e de idioma (idiomas que os autores dominam) resultaram na leitura de 18 artigos e posteriormente em mais seis, a partir de referências dos primeiros.

Palavras-chave: Diabetes Mellitus; Diabetes Ketoakidosis (DKA), Non Ketotic Hyperglycemic Hyperosmolar Sindrome (NKHHS), Hypoglycemia (HYPO), Lactoacidosis (LAK) e Family Practice.

*Médica de Família Centro Saúde de Vila Franca Xira ${ }^{* *}$ Chefe de Serviço de Diabetologia do Hospital Santa Maria

\section{INTRODUCÃO}

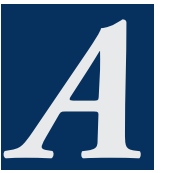

s complicações agudas da diabetes (hipoglicemia, cetoacidose diabética, síndrome hiperglicémico hiperosmolar e lactoacidose) são causa de recorrência à urgência e internamento e estão associadas, na maioria dos casos, a intercorrências médicas e/ou falhas terapêuticas. ${ }^{1-3}$

A pessoa com diabetes apresenta-se sempre como um potencial problema diagnóstico, mesmo com sintomas aparentemente ligeiros, como febrícula, cansaço, tonturas ou sonolência. O Médico de Família (MF) deve, perante um diabético descompensado, ter sempre subjacente a suspeita destas situações, avaliar de forma cuidada, para poder detectar sinais, mesmo não muito expressivos, de hipo ou hiperglicemia, e possibilitar a prevenção atempada de uma complicação aguda cuja rapidez de evolução e agravamento até ao coma é, ainda hoje, causa evitável de morte. ${ }^{4}$

A prevenção destas complicações passa, sobretudo, pela educação terapêutica, com a capacitação da pessoa com diabetes para tomar as decisões adequadas ao seu controlo, em particular durante doenças intercorrentes, ${ }^{5} \mathrm{e}$ pela identificação de pessoas de maior risco (exemplo, idosos em lares).

\section{CRISES HIPERGIICÉmicas AgUdAS}

A Cetoacidose Diabética (DKA) e o Síndrome Hiperglicémico Hiperosmolar (NKHHS) são duas complicações agudas graves da diabetes, cuja morbilidade e mortalidade se mantêm elevadas, apesar dos avanços terapêuticos actuais, e para cuja prevenção e detecção precoce o MF deve estar preparado.

A DKA, apesar de mais frequente na diabetes tipo 1 e nos jovens, pode surgir também na diabetes tipo 2 e na diabetes inaugural do adulto, sendo que $80 \%$ dos casos recorrentes correspondem a cerca de $20 \%$ dos doentes, cuja identificação se torna particularmente importante em Medicina Geral e Familiar (MGF), por se tratarem de casos/família problema, onde urge actuar de forma proactiva na prevenção. ${ }^{5}$

A mortalidade por DKA é < 5\% e ronda os $11 \%$ no NKHHS, ${ }^{6}$ não parecendo ser alterada pelo tipo de hospital de tratamento ou pela especialidade do médico, desde que sejam seguidas as orientações técnicas terapêuticas adequadas, estando relacionada, na maioria dos casos, com a doença precipitan- 


\section{DOSSIER}

te da situação. ${ }^{2,6,7}$

Apesar da patogénese da DKA ser melhor compreendida que o NKHHS, o mecanismo subjacente em ambas as situações resulta da combinação de uma elevação das hormonas de contra-regulação (glucagon, catecolaminas, cortisol e hormona de crescimento) e de uma concentração baixa efectiva de insulina, pelo que ambas as situações se assemelham a manifestações extremas da alteração do metabolismo dos hidratos de carbono. Apesar de muitos doentes apresentarem um quadro clínico sobreposto, cada uma destas condições pode surgir em formas clínicas relativamente puras. ${ }^{4,6}$

\section{Cetoacidose Diabética (DKA)}

A atenção precoce aos sintomas de DKA permite o diagnóstico, quer na diabetes inaugural, quer em diabéticos conhecidos em fase de descompensação. ${ }^{7}$ Os sintomas podem desenvolver-se em horas (Guadro I) e são, por vezes, a primeira manifestação da diabetes tipo 1 , mas surgem mais frequentemente em situações de descompensação da diabetes, ocasionada por uma situação de falha na toma de insulina ou numa situação de stress, com o aumento consequente das necessidades de insulina (exemplo, doença intercorrente). ${ }^{2,9}$

A dor abdominal, as náuseas e os vómitos podem ser intensos e simular uma situação de abdómen agudo, tornando importante a utilização de exames complementares, mesmo na primeira linha do atendimento urgente (glicemia e bioquímica urinária determinadas com tiras reagentes), para além da avaliação dos sinais físicos (tensão arterial, frequência cardíaca e respiratória). Devem ser considerados os quadros diagnósticos diferenciais com as situações de acidose metabólica e produção aumentada de corpos cetónicos (Quadro II) ao mesmo tempo que as causas desencadeantes mais frequentes (Quadro III).

\section{Patogénese}

Num indivíduo hidratado com diabetes anteriormente bem controlada, a falta efectiva de insulina (défice relativo ou absoluto) associa-se ao aumento de glucagon, catecolaminas, hormona de crescimento e cortisol. A hiperglicemia ocorre como resultado simultâneo de uma gluconeogénese aumentada, de uma glucogenólise acelerada e de uma deficiente utilização da glucose nos tecidos periféricos, desencadeando uma cetose ligeira.

Se houver seguimento de vómitos ou em situações de stress, a desidratação

QUADRO I

\begin{tabular}{|c|c|}
\hline \multicolumn{2}{|c|}{ SINTOMAS E SINAIS DA DKA E DO NKHSS } \\
\hline Sintomas & Sinais \\
\hline $\begin{array}{l}\text { - Astenia progressiva até letargia } \\
\text { - Anorexia } \\
\text { - Náuseas e vómitos (DKS) } \\
\text { - Presença de «Polis» (poliúria e polidipsia) } \\
\text { - Dor abdominal - também torácica ou outras } \\
\text { localizações - (DKS) } \\
\text { - Boca seca (sem saliva) }\end{array}$ & $\begin{array}{l}\text { - Temperatura normal ou baixa (se alta suspeitar } \\
\text { de infecção subjacente) } \\
\text { - Polipneia profunda (Kussmaul) (DKS) } \\
\text { - Desidratação: } \\
\text { - } 5 \% \text {, se perda do turgor, mucosas secas e taquicardia } \\
-10 \% \text {, se revascularização capilar } \geq 3 \text { " e olhos encovados } \\
->10 \% \text {, se prega cutânea, hipotensão, oligúria e choque } \\
\text { - Alterações da consciência } \\
\text { - Perda de peso }\end{array}$ \\
\hline
\end{tabular}


1. Cetoacidose alcoólica - neste caso a história de jejum prolongado e de vómitos intensos, com valores normais ou moderadamente elevados de glicemia, associada à ingestão aguda ou crónica de álcool, fará a suspeita diagnóstica;

2. Cetoacidose da fome - em geral ligeira, pode ocorrer após fome prolongada;

3. Outras causas de acidose metabólica com aumento da produção de ácido láctico

1. Acidose láctica secundária a falência respiratória ou circulatória

2. Acidose láctica associada a outras alterações

3. Acidose láctica induzida por drogas e toxinas

4. Acidose láctica devida a defeitos enzimáticos

5. Acidose pós envenenamento por salicilatos, metanol e glicol de etileno.

\section{QUADRO III}

\section{FACTORES PRECIPITANTES DA DKA}

- Administração inadequada de insulina (défice absoluto ou relativo) - causa mais frequente nos adolescentes e nas classes sociais mais baixas;

- Infecções (pneumonia, tracto urinário, gastroenterite, apendicite, colecistite, abcessos, sepsis);

- Enfarte isquémico (miocárdio, cerebral, mesentérico, periférico);

- Drogas (cocaína);

- Fármacos que agravam a diabetes (corticoterapia, psicotrópicos atípicos);

- Gravidez.

pode agravar o quadro de hiperglicemia que, por sua vez, causa diurese osmótica pela glicosúria, com perda de água e electrólitos, hipovolémia, desidratação e diminuição do filtrado glomerular.

A razão insulina/glucagon, já alterada na diabetes, aumenta e surgem modificações no equilíbrio entre enzimas chaves do metabolismo, com o consequente aumento da produção de glucose pela neoglucogénese hepática.

A desidratação progressiva, com hipovolemia e acidemia, condiciona a perturbação da consciência e também da perfusão tecidular, nomeadamente cardíaca, podendo determinar o choque.

\section{Tratamento $^{10,11}$}

A DKA é um estado pró-inflamatório, com produção de corpos oxidativos, que reverte com o restabelecimento dos níveis de insulina e glicemia normais. ${ }^{12}$
As alterações metabólicas desenvolvem-se num largo espectro, que se inicia numa acidose ligeira com hiperglicemia moderada, mas que pode progredir rapidamente para formas mais graves, pelo que importa o tempo do seu correcto diagnóstico (Quadros VI e V) e tratamento imediato (Quadro VI), com contacto e referenciação a uma unidade de cuidados especializados, sobretudo numa pessoa com quadro sugestivo e com vómitos.

No Centro de Saúde: Avaliar o estado do doente e tentar diagnosticar a causa subjacente da descompensação.

1. Doente diabético descompensado com cetonúria sem vómitos

A correcção da desidratação deve ser promovida através da ingestão de líquidos. A correcção da cetose faz-se através do aporte de insulina regular subcutânea, segundo os valores da glicemia, a cada quatro horas, até cetona nega- 


\section{DOSSIER}

Confirmar o diagnóstico de imediato

- Glicemia $\geq 200 \mathrm{mg}$ ( $11 \mathrm{mmol} / \mathrm{L})$

- Glicosúria e cetonúria positivas $(++,+++)$; se o diabético não consegue urinar testar na secreção lacrimal ou, se tiver acesso, determinar no sangue capilar (cetonemia $>3 \mathrm{mmol} / \mathrm{L}$ )

(Se apenas + com glicemia $<200$ suspeitar de hipoglicemia). Na dúvida referenciar imediatamente.

0 laboratório poderá ainda mostrar:

(Como se trata de uma emergência deve actuar-se perante a suspeita e não esperar por testes de laboratório)

- Leucocitose - constante (devida à acidose) e neutrofilia - se houver infecção

- Acidose - mais tarde acidemia

- $\mathrm{K}^{+}$- normal ou, mais frequentemente, elevado devido à saída do potássio celular pela acidose

- $\mathrm{Na}^{+}$- baixo, face à modesta concentração osmolar (se marcada descida, artefacto por hipertrigliceridemia ou devido aos vómitos intensos), mas pode estar normal ou elevado.

\begin{tabular}{|c|c|c|c|c|}
\hline \multicolumn{5}{|c|}{ QUADRO V } \\
\hline \multicolumn{5}{|c|}{ VALORES LABORATORIAIS NA DKA E NO NKHHS } \\
\hline & \multicolumn{3}{|c|}{ Cetoacidose Diabética } & \multirow{2}{*}{$\begin{array}{c}\text { Síndrome } \\
\text { Hiperglicemico } \\
\text { Hiperosmolar }\end{array}$} \\
\hline & Ligeira & Moderada & Grave & \\
\hline Glicemia plasmática & $\begin{array}{l}>250 \mathrm{mg} / \mathrm{dl} \\
(>13,9 \mathrm{mmL} / \mathrm{L})\end{array}$ & $\begin{array}{l}>250 \mathrm{mg} / \mathrm{dl} \\
(>13,9 \mathrm{mmL} / \mathrm{L})\end{array}$ & $\begin{array}{l}>250 \mathrm{mg} / \mathrm{dl} \\
(>13,9 \mathrm{mmL} / \mathrm{L})\end{array}$ & $\begin{array}{l}>600 \mathrm{mg} / \mathrm{dl} \\
(>33,3 \mathrm{mmL} / \mathrm{L})\end{array}$ \\
\hline Cetonuria & ++ & +++ & ++++ & + \\
\hline Cetonemia & $3-5 \mathrm{mmol} / \mathrm{L}$ & $5-12 \mathrm{mmol} / \mathrm{L}$ & $>12 \mathrm{mmol} / \mathrm{L}$ & $<5 \mathrm{mmol} / \mathrm{L}$ \\
\hline Bicarbonatos & $15-18 \mathrm{mEq} / \mathrm{L}$ & $10<15 \mathrm{mEq} / \mathrm{L}$ & $<10 \mathrm{mEq} / \mathrm{L}$ & $>15 \mathrm{mEq} / \mathrm{L}$ \\
\hline Osmolaridade sérica efetiva* & Variável & Variável & Variável & $>320 \mathrm{~m} 0 \mathrm{sm} / \mathrm{Kg}$ \\
\hline pH arterial & $7,25-7,30$ & $7,00-7,25$ & $<7,00$ & $>7,30$ \\
\hline Ureia & $\uparrow$ & $\uparrow$ & $\uparrow \uparrow$ & $\uparrow \uparrow \uparrow$ \\
\hline TG & $\uparrow$ & $\uparrow$ & $\uparrow$ & $\uparrow$ \\
\hline Leucocitos & $\uparrow$ & $\uparrow$ & $\uparrow$ & $\uparrow$ \\
\hline Água (défice) & $5 \%$ & $5-10 \%$ & $15 \%$ & $>15 \%$ \\
\hline Intervalo anionico ${ }^{* *}$ & $>10 \mathrm{mEq} / \mathrm{L}$ & $>12 \mathrm{mEq} / \mathrm{L}$ & $>12 \mathrm{mEq} / \mathrm{L}$ & $<12 \mathrm{mEq} / \mathrm{L}$ \\
\hline \multicolumn{3}{|c|}{ *Formula de cálculo: $2 x[(\mathrm{Na} \mathrm{mEq} / \mathrm{L}+\mathrm{G} \mathrm{mg} / \mathrm{dl}) / 18]^{2,9}$} & \multicolumn{2}{|c|}{ Normal $280-295 \mathrm{~m} 0 \mathrm{sm} / \mathrm{kg}$} \\
\hline ** Formula de cálculo: [Na- (C & & & & \\
\hline
\end{tabular}

tiva na urina. Após a correcção e tratamento da intercorrência desencadeante deve programar-se uma consulta de revisão e promoção das medidas preventivas.

\section{Doente diabético com quadro de ceto-} se, vómitos e desidratação

- Contactar imediatamente a equipa de urgência hospitalar mais próxima, informando a hora e os valores obtidos na monitorização clínica do doente.

- A correcção da perda de fluidos deve iniciar-se imediatamente, utilizando, se a glicemia for > $250 \mathrm{mg} / \mathrm{dl}$ (13,9 $\mathrm{mmol} / \mathrm{L}$ ), soro fisiológico (soro isotónico salino) e soro dextrosado a 5\%, 


\section{TRATAMENTO DA CRISE COM CETOSE}

Tratamento da crise com cetose

1. Identificação e tratamento da causa;

2. Se doente não nauseado, dar IL de líquidos (água, caldo de cozer legumes, chá) a beber lentamente durante cada uma-duas horas; se nauseado usar solutos intravenosos ou referenciar;

3. Se glicemia $<250 \mathrm{mg} / \mathrm{dl}$ manter o tratamento habitual com vigilância das glicemias e da cetonúria mais frequentes;

4. Se Glicemia $>250 \mathrm{mg} / \mathrm{dl}$ dar 10Ul de insulina regular (intramuscular na primeira vez, se suspeita de desidratação) subcutânea cada duas horas até glicemia $<250 \mathrm{mg} / \mathrm{dl}$ e diminuição da cetonúria (esta pode levar mais tempo a desaparecer).

se a glicemia for < $250 \mathrm{mg} / \mathrm{dl}$ (13,9 $\mathrm{mmol} / \mathrm{L})$, para expansão do volume intravascular e restabelecimento da perfusão renal.

- Se necessário, administrar insulina regular, subcutânea ou intramuscular, consoante a glicemia.

Na Unidade Hospitalar (segundo o protocolo da mesma):

- Iniciar tratamento com insulina intravenosa, usando preferencialmente uma bomba infusora, numa perfusão de 7-10UI por hora, até a glicemia alcançar valores < $50 \mathrm{mg} / \mathrm{dl}$ (13,9mmol/L). Nessa altura diminuir a perfusão para 2 UI por hora.

- Os electrólitos devem ser monitorizados logo que possível e a cada quatro horas. Todos os electrólitos [sódio $\left(\mathrm{Na}^{+}\right)$, cloretos (HCL), fósforo (P) e magnésio $\left(\left[\mathrm{Mg}^{++}\right)\right]$estão diminuídos na DKA mas os seus valores séricos não reflectem o seu valor total devido à hiperglicemia e desidratação. Os valores de creatinina sérica associados aos de ureia reflectem de forma mais fiável a depleção hídrica intravascular. $\mathrm{O} \mathrm{Na} \mathrm{Na}^{+}$encontra-se reduzido como consequência da hiperglicemia, pelo que um valor de $\mathrm{Na}^{+}$normal pode fazer suspeitar de uma desidratação mais profunda. Apesar do défice corporal total de $\mathrm{K}^{+}$, o seu valor sérico pode revelar-se no início normal ou ligeiramente aumentado secundariamente à acidose.

- Se a função renal estiver assegurada e os niveis de $\mathrm{K}^{+}$forem $<5,5 \mathrm{mEq} / \mathrm{L}$, deve administrar-se cloreto de potássio na perfusão.

- A acidose pode corrigir-se apenas pelo tratamento com a insulina. A administração de bicarbonato não está recomendada, excepto se muito severa (ex.: $\mathrm{pH}<6,9, \mathrm{HCO}_{3}<5$ mEq/ /L), pois pode provocar edema pulmonar, insuficiência respiratória ou hipercaliémia.

- Em indivíduos com compromisso renal ou cardíaco deve monitorizar-se, para além destas funções, a osmolaridade sérica e o estado mental a fim de evitar a sobrecarga hídrica iatrogénica.

- Perante uma situação de coma, a entubação e a manutenção das vias aéreas devem ser consideradas, tal como a entubação nasogástrica e a algaliação.

A DKA na criança é um diagnóstico mais dificil e a sua potencial letalidade e complicação por edema cerebral $^{13}$ obriga a encaminhar o mais brevemente possivel para um centro especializado, com contacto prévio, devendo iniciar-se a monitorização e hidratação imediatas. $^{10}$

Considera-se resolvida a crise aguda de DKA quando a glicemia retorna a valores $<200 \mathrm{mg} / \mathrm{dl}$, a cetonúria é nega- 
tiva (3-hidroxibutirato $<1 \mathrm{mmol} / \mathrm{L}$ ), o $\mathrm{pH}$ $>7,3$ e o bicarbonato sérico $\geq 18 \mathrm{mEq} /$ /L. No entanto, deve continuar-se a suplementação hídrica enquanto não se restabelecer a via oral e passar a administração de insulina endovenosa a subcutânea. Quando a via oral estiver restabelecida, deve rever-se o esquema de insulina subcutânea.

A alta hospitalar destes doentes e a consulta posterior no Centro de Saúde devem ser programadas, para reforço das medidas educativas de capacitação do diabético, em particular toda a informação sobre as medidas preventivas a tomar nas intercorrências, diagnóstico precoce e correcção da cetose.

O diabético deve ser ensinado e incentivado a fazer a pesquisa de cetonúria na primeira micção da manhã sempre que suspeitar de desequilíbrio (glicemia $>300 \mathrm{mg} / \mathrm{dl} \mathrm{ou}>16,5 \mathrm{mmol} / \mathrm{L}$ ). No caso de cetonúria deve efectuar de imediato a sua correcção. A prevenção deste problema nos dias de doença ou maior stress é muito importante e deve ser discutida com o diabético com regularidade.

O tratamento da DKA tem como objectivo a correcção da desidratação, reverter a cetose e a acidose, reduzir a glicemia para valores normais, corrigir os electrólitos e as perdas hídricas, ao mesmo tempo que se identifica e trata a causa desencadeante subjacente. ${ }^{14}$

\section{SíndROME HIPERGIICÉMICO HIPEROSMOLAR (NKHHS)}

Este síndrome define-se por uma hiperglicemia superior a $600 \mathrm{mg} / \mathrm{dl}$ associada a uma osmolaridade sérica superior a $320 \mathrm{mOsm} / \mathrm{L}$, podendo ou não coexistir com cetoacidose (geralmente ligeira) e acidose láctica.

Na maioria dos casos o factor precipitante é uma doença concomitante e os seus factores predisponentes são a idade avançada, nível de dependência, sexo feminino, internamento num lar de idosos e infecções, ocorrendo um elevado número de casos em doentes sem história anterior de diabetes. A incidência e a mortalidade desta situação são suficientemente elevadas para que um MF se tenha de confrontar com o seu diagnóstico e tratamento numa unidade de urgência ou mesmo no domicilio. ${ }^{15}$

Os sintomas (Quadro I) desenvolvem-se de forma mais lenta (dias a semanas), com poliúria, perda de peso e diminuição da ingestão oral, culminando em alterações da consciência, que vão da letargia ao coma. O exame clínico revela profunda desidratação, hipotensão, taquicardia e alteração da consciência, na ausência de náuseas, vómitos, dores abdominais e polipneia.

\section{Patogénese}

As causas subjacentes são um défice de insulina e uma inadequada hidratação. A falta efectiva de insulina aumenta a produção de glucose, através da estimulação das neoglucogénese e glucogenólise, ao mesmo tempo que altera a captação da glucose no músculo-esquelético e tecido adiposo, da mesma forma que na DKA. A hiperglicemia induz diurese osmótica pela glicosúria, desencadeando desidratação, que é agravada pela inadequada ingestão de líquidos. Apesar de não inteiramente compreendida, a ausência de cetona pode dever-se a uma deficiência de insulina menos grave que na DKA, com níveis menores de hormonas de contra regulação e ácidos gordos livres (FFA), com menor capacidade do figado em produzir os corpos cetónicos.

\section{Tratamento}

No NKHHS, tal como na DKA, o objectivo é expandir o volume intravascular através da hidratação, pelo que se recomenda a perfusão com $1 \mathrm{~L}$ de solução salina a $0,9 \%$ na primeira meia-hora, que deve ser repetida caso o doente per- 
maneça hipotenso. No entanto, ao contrário da DKA, existe um défice hídrico marcado, pelo que se deve ter o cuidado de fazer a correcção gradualmente a fim de evitar o desequilíbrio osmótico entre o plasma e o sistema nervoso central, com o consequente edema cerebral. Os fluidos intravenosos devem ser alterados para solução salina a $0,45 \%$, assim que a perfusão vascular se apresentar razoável, a uma frequência de 300-500 ml/hora, ajustada às funções renais e cardíacas.

A monitorização da glicemia deve efectuar-se a cada uma-duas horas e a dos electrólitos a cada quatro horas. Deve iniciar-se a terapia com insulina intravenosa num bolus de 5-10UI mantendo, após estabilização, 3-7UI/hora em perfusão.

A glicemia diminui e o $\mathrm{Na}^{+}$aumenta, apenas com a hidratação, pelo que, assim que a glicemia for $<250 \mathrm{mg} / \mathrm{dl}$ o soluto deve passar a dextrose $5 \%$ em água, caso haja aumento do $\mathrm{Na}^{+}$( > 155 $\mathrm{mEql} / \mathrm{l}$ ), ou em soluto salino, se normal.

A hipocaliemia é menos severa que na DKA mas pode ser necessário adicionar KCL na perfusão para repor os seus niveis.

A monitorização da osmolaridade e do estado de consciência é essencial em doentes com função renal e/ou cardíaca comprometidas e para evitar a sobrecarga hídrica iatrogénica.

O tratamento com bicarbonato só é necessário se a situação se complicar com lactoacidose (lactacidemia $\geq 2,0$ $\mathrm{mmol} / \mathrm{L}$ e $\mathrm{pH} \leq 7,3$ sem cetonúria), situação rara, geralmente precipitada pelo uso concomitante de metformina em indivíduos com função renal comprometida (clearance creatinina $<30$ $\mathrm{ml} / \mathrm{min})$.

A mortalidade neste sindrome é elevada e deve-se sobretudo à presença de comorbilidade e de doença precipitante grave. ${ }^{6}$ A prevenção desta complicação passa sobretudo pela vigilância dos grupos mais vulneráveis (idosos, sobre- tudo dependentes) e treino dos cuidadores.

\section{Hipoglicemia}

A hipoglicemia é a alteração metabólica aguda mais frequente que os doentes tratados com fármacos antidiabéticos hipoglicemiantes, incluindo a insulina, podem enfrentar, e define-se por descida excessiva de glicemia para valores inferiores a $50 \mathrm{mg} / \mathrm{dl}(2,75 \mathrm{mmol} /$ /L). ${ }^{16} \mathrm{~A}$ hipoglicemia traduz-se num conjunto de sintomas e sinais de natureza reactiva-vegetativa e/ou por carência de glicose na célula nervosa, que melhora com a administração de glicose, mas que caso não tratada pode conduzir a lesão neurológica e morte.

Os sintomas de hipoglicemia (Figura 7) variam de pessoa para pessoa e é importante que cada um saiba reconhecer os mais frequentes. Se a hipoglicemia ocorre repetidamente pode iniciar um ciclo vicioso de reacções fisiológicas que mascaram ou diminuem a capacidade de detectar os primeiros sinais de aviso, motivando episódios de hipoglicemia severa. ${ }^{17}$ Os sintomas descritos

\section{QUADRO VII}

SINTOMAS MAIS FREQUENTES DE HIPOGLICEMIA

- Sudorese

- Tremores

- Incapacidade de se concentrar

- Confusão

- Boca seca

- Visão turva

- Fome

- Ansiedade

- Cefaleias

- Dificuldade em andar

- Palpitações

- Parestesias peribucais

- Dificuldade em falar

- Tonturas

- Alterações do comportamento

- Náuseas 
pela pessoa estão relacionados com a rapidez da descida da glicemia e os seus efeitos no cérebro e sistema nervoso autonómico, mediados pelas hormonas e neurotransmissores. Numa descida rápida da glicemia, a estimulação da adrenalina e do glucagon provoca um sindrome vegetativo, com alterações da frequência cardíaca (taquicardia e palpitações), ansiedade, vasoconstrição com palidez, sudorese fria (por vezes profusa), tremores ou sensação de tremor interior e fome intensa. Se a instalação do quadro é mais lenta predominam os sintomas neuroglicopénicos, como cefaleias, tonturas, parestesias peribucais e da lingua, alterações da visão (turva), lentificação do raciocínio, alteração da memória, do humor e do comportamento, alteração dos movimentos finos e da fala, quadros de hemiparésia, confusão mental, convulsões e mesmo coma. O diagnóstico deve ser efectuado com rapidez assim como o seu tratamento. A pesquisa de glicemia capilar deve ser posterior ao tratamento, na suspeita clínica. O diagnóstico diferencial deve ser efectuado entre as causas de hipoglicemia de jejum e as de pós-prandial ou reactiva (Quadro VIII).

A hipoglicemia é mais comum nos diabéticos tipo 1 ou nos diabéticos tipo 2 em tratamento intensivo com antidiabéticos orais e/ou com insulina, sobretudo naqueles com maior duração de diabetes e com maior necessidade de insulina exógena para um melhor controlo. A hipoglicemia grave é mais frequente em crianças $^{18}$ e idosos, sendo ainda causa de recorrência aos serviços de urgência e internamento. A hipoglicemia causada pelas sulfonilureias (SUF), sobretudo pelas mais antigas, é mais frequente e recidivante, ${ }^{19}$ pelo que os doentes medicados com estes fármacos devem ser ensinados a prevenila e a tratá-la com atenção. Este tipo de antidiabético oral pode, eventualmente, ser substituído por outras SUF ou pelas nateglinidas, com menor risco de hipoglicemia, ${ }^{20}$ especialmente em idosos e diabéticos com neuropatia autonómica.

A terapêutica com insulina na diabetes tipo 2 é muito frequentemente retardada pelo medo da hipoglicemia, sobretudo ao nível dos cuidados de saúde primários. ${ }^{21}$ A utilização de insulina neutral protamina Hagedorn (NPH) como insulina basal associada a terapêutica com antidiabéticos orais pode ser começada em titulação lenta ao deitar com menos hipoglicemias. ${ }^{22,23}$ Existem actualmente, para os casos individuais de maior risco, análogos de insulinas (glargina e levemir) com menor risco de hipoglicemia. ${ }^{24,25}$

A hipoglicemia nocturna é uma preocupação para os doentes e profissionais devido à possibilidade daquela ser mascarada pelo sono. Este problema é de dificil estudo e exige, por vezes, a monitorização de valores nocturnos.

As hipoglicemias, quer as autotratadas quer as heterotratadas (que exigem tratamento por terceiros) são mais frequentes nos diabéticos de tipo 1 com diabetes de maior duração do que nos diabéticos tipo 2 tratados com SUF. ${ }^{26}$

As hipoglicemias devem ser assinaladas nos registos de autovigilância. Este exercício é fundamental no processo de aprendizagem do autocontrolo e este deve ser analisado em conjunto com a equipa de profissionais, revendo a alimentação (sobretudo a ingestão de hidratos de carbono), o exercício e as doses dos fármacos, para além de eventuais intercorrências ou interacções medicamentosas. A base da educação terapêutica na hipoglicemia é o reforço da prevenção, reconhecimento precoce dos sintomas e tratamento (Quadro XIX) atempado pelo diabético e seus cuidadores. Os doentes devem ser ensinados a reconhecer os seus sintomas, a tratar de imediato a situação, a identificar e corrigir o factor desencadeante, usando o erro como factor de aprendizagem. ${ }^{27} \mathrm{O}$ tratamento inicial da hipo- 


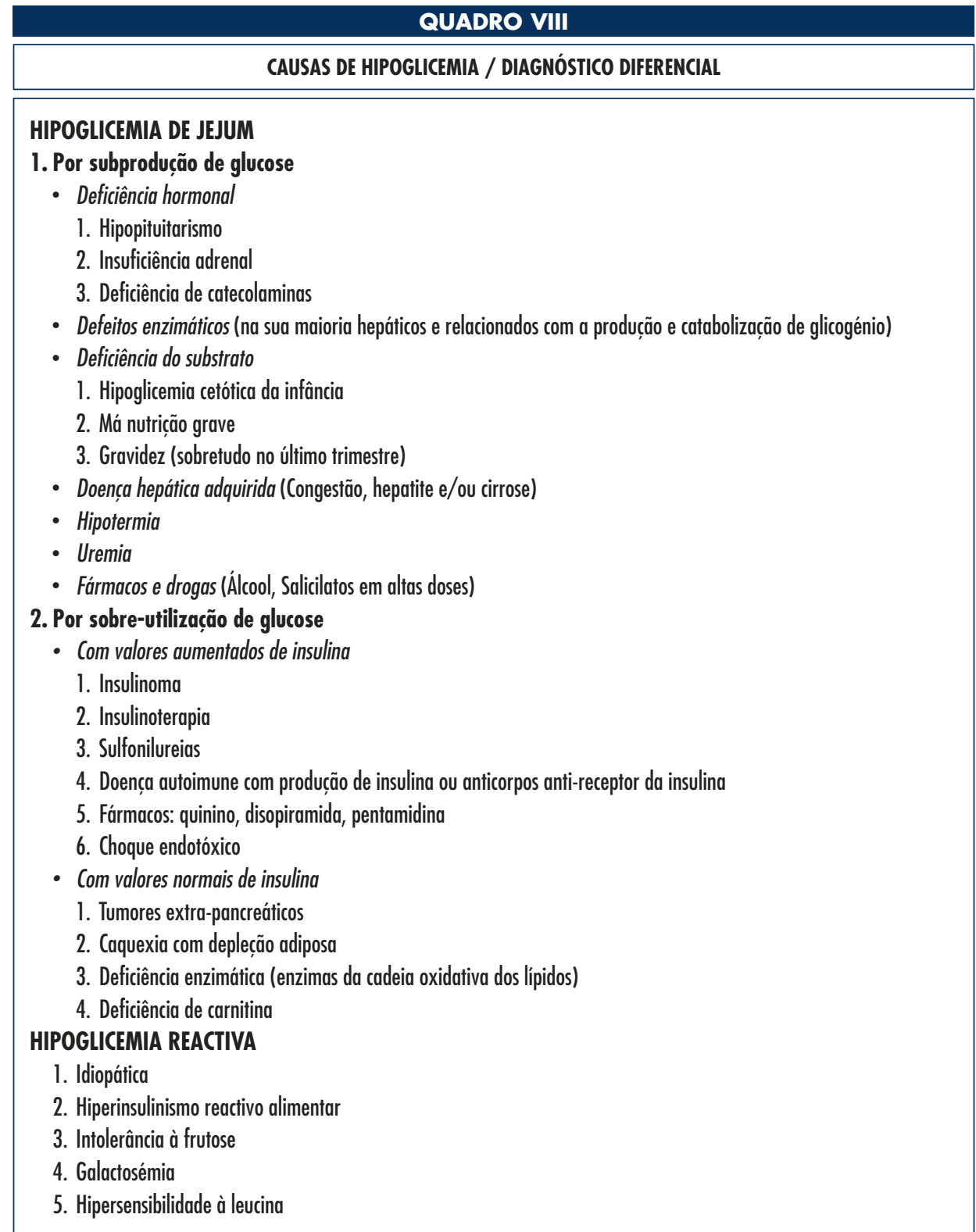

glicemia com alimentos de digestão mais lenta induz a perda de sensibilidade aos sintomas vegetativos, pelo que o diabético deve ser ensinado a ingerir sacarose ou glucose preferencialmente.

\section{CONCLUSÃO}

As complicações agudas da diabetes são preveníveis pela educação terapêutica continuada da pessoa com diabetes e dos seus cuidadores mas mantêm--se um problema actual, sobretudo nas crises de hiperglicemia com vómitos e desidratação, pela sua elevada incidência e mortalidade. O MF deve estar preparado para efectuar o diagnóstico e o tratamento atempados e contactar com a equipa dos cuidados secundários, sem- 


\section{DOSSIER}

1. Doente consciente - o controlo imediato faz-se com a ingestão de solução fraca de sacarose (2 pacotes de açúcar em água) ou glicose, repetindo, se necessário, até ao desaparecimento dos sintomas.

2. Doente agitado ou negativista - administrar uma papa de açúcar feita com adição de umas gotas de água.

3. Deglutição impossível - administrar glucose hipertónica a 20 \% IV até à recuperação da consciência (na impossibilidade da sua administração fazer lmg de glucagon im). Nota: baixo efeito do glucagon no paciente alcoolizado ou malnutrido.

- Se após 100 cc não houver retoma da consciência fazer uma determinação da glicemia capilar

- Se $\leq \mathbf{2 0 0 ~ m g / d l , ~}(11 \mathrm{mmo} / \mathrm{L})$ continuar administração de glicose hipertónica até obter este valor e manter em vigilância.

- Se $>200 \mathrm{mg} / \mathrm{dll}$, trata-se de um coma prolongado com edema cerebral que exige internamento hospitalar urgente.

- No caso de doente a fazer inibidores da alfa-glucosidase (acarbose) deve tomar glucose e não sacarose para evitar os atrasos de absorcão.

- Só depois de corrigida a hipoglicemia e a consciência deve ser ingerida pequena refeição contendo hidratos de carbono de absorção lenta.

- Se a causa for 0 uso de sulfonilureias (em particular as mais antigas) o doente deve ser mantido em vigilância durante 24h, pois a acção destas é longa e pode haver recidiva

pre que necessário e o mais breve possivel, assim que suspeitar de qualquer uma das situações.

\section{REFERÊNCIAS BIBLIOGRÁFICAS}

1. Chung ST, Perue GG, Johnson A, Younger N, Hoo CS, Pascoe RW, et al. Predictors of hyperglycaemic crises and their associated mortality in Jamaica. Diabetes Res Clin Pract 2006 Aug; 73 (2):184-90.

2. Kitabchi AE, Umpierrez GE, Murphy MB, Kreisberg RA. Hyperglycemic crises in adult patients with diabetes: a consensus statement from the American Diabetes Association. Diabetes Care 2006 Dec; 29 (12): 2739-48.

3. Kitabchi AE, Umpierrez GE, Murphy MB, Barrett EJ, Kreisberg RA, Malone JI, et al. Management of hyperglycaemic crises in patients with diabetes. Diabetes Care 2001 Jan; 24 (1): 131-53.

4. Rosenbloom AL. Hyperglycemic crises and their complications in children. J Pediatr Endocrinol Metab 2007 Jan; 20 (1) 5-18.

5. Booth GL, Hux JE, Fang J, Chan BT. Time trends and geographical disparities in acute complications of diabetes in Ontario, Canada. Diabetes Care 2005 May; 28 (5): 1045-50.

6. MacIsaac RJ, Lee LY, McNeil KJ, Tsalamandris C, Jerums G. Influence of age on the presentation and outcomes of acidotic and hyperosmolar diabetic emergencies. Intern Med Journal 2002 Aug; 32 (8): 379-85.

7. Phillips LS, Ziemer DC, Doyle JP, Barnes CS, Kolm P, Branch WT, et al. An endocrinologist-supported intervention aimed at providers improves diabetes management in a primary care site: improving primary care of African Americans with diabetes (IPCAAD) 7. Diabetes Care 2005 Oct; 28 (10): 2352-60.

8. Wolfsdorf J, Glaser N, Sperling MA. Diabetic ketoacidosis in infants, children, and adolescents: a consensus statement from the American Diabetes Association. Diabetes Care 2006 May; 29 (5): 1150-9.

9. Powers AC. Diabetes mellitus acute complications. In: Kasper DL, Braunwald E, Fauci A, Hauser S, Longo D, Jameson L, editors. Harrison's Principles of Internal Medicine. 16th ed. New York: McGraw-Hill; 2005. p. 2158-63.

10. Faro T, Parreira JM. Terapêutica das descompensações agudas da diabetes: a cetoacidose diabética e a sindrome hiperosmolar hiperglicémica. In: Duarte R, editor. Diabetologia clínica. $3^{\text {a }}$ ed. Lisboa: Lidel; 2002. p. 209-17.

11. Genuth SM, Gebhart SS, Friedman EA, Jovanovic L, Gerich JE, Tamborlane WV, et al. Special Situations. In: Cefalu WT, Gerich JE, LeRoith D, editors. The Cadre Handbook of Diabetes Management. New York: Medical Information Press; 2004. p. 169-220. Disponível em: URL: http://www.cadre-diabetes.net/s_ 
cadre_book.asp [acedido em 30/10/2007].

12. Clement S, Braithwaite SS, Magee MF, Ahmann A, Smith EP, Schafer RG, et al. Management of diabetes and hyperglycaemia in hospitals. Diabetes Care 2004 Feb; 27 (2): 553-91.

13. Trachtenbarg DE. Diabetic ketoacidosis. Am Fam Physician 2005 May 1;71(9): 1705-14.

14. Charfen MA, Fernández-Franckelton M. Diabetic ketoacidosis. Emerg Med Clin North Am 2005 Aug; 23 (3): 609-28.

15. Wachtel TJ. The diabetic hyperosmolar state. Clin Geriatr Med 1990 Nov; 6 (4): 797 $-806$.

16. Andre O, Gonçalves J. hipoglicemia. In: Duarte R, editor. Diabetologia clínica. $3^{\mathrm{a}}$ ed. Lisboa: Lidel; 2002. p. 219-27.

17. Cefalu CA, Cefalu WT. Controlling hypoglycaemia in type 2 diabetes: which agent for which patient? J Fam Pract 2005 Oct; 54 (10): 855-62.

18. Bulsara MK, Holman CD, Davis EA, Jones TW. The impact of a decade of changing treatment on rates of severe hypoglycemia in a population-based cohort of children with type 1 diabetes. Diabetes Care 2004 Oct; 27 (10): 2293-8.

19. Gallego R. Terapêutica oral da diabetes tipo 2. Rev Port Clin Geral 2005 Nov-Dec; 21 (6): 575-84.

20. Gangji AS, Cukierman T, Gerstein HC, Goldsmith $\mathrm{CH}$, Clase CM. A systematic review and meta-analysis of hypoglycemia and cardiovascular events: a comparison of glyburide with other secretagogues and with insulin. Diabetes Care 2007 Feb; 30 (2): 389-94.

21. Jeavons D, Hungin AP, Cornford CS. Patients with poorly controlled diabetes in primary care: healthcare clinicians' beliefs and attitudes. Postgrad Med J 2006 May; 82 (967): 347-50.

22. Yki-Järvinnen H. Combination therapies with insulin in type 2 diabetes. Diabetes Care 2001 Apr; 24 (4): 758-67.

23. Yki-Järvinnen H, Kauppila M, Kujansuu E, Lahti J, Marjanen T, Niskanen L, et al. Comparison of insulin regimens in patients with non-insulin-dependent diabetes mellitus. N Engl J Med 1992 Nov 12; 327 (20): 1426-33.

24. Yki-Jarvinen H. Insulin therapy in type 2 diabetes: role of the long-acting insulin glargine analogue. Eur J Clin Invest 2004 Jun; 34 (6): 410-6.

25. Bacelar C. Terapûtica com insulina na diabetes tipo 2. Rev Port Clin Geral 2005; 21 : 619-23.

26. UK Hypoglyceamia Study Group. Risk of hypoglycaemia in types 1 and 2 diabetes: effects of treatment modalities and their duration. Diabetologia 2007 Jun; 50 (6): 1140-7.

27. Singh R. How a series of errors led to a recurrent hypoglycemia. J Fam Pract 2006 Jun; 55 (6): 489-97.

\section{ACUTE COMPLICATIONS OF DIABETES MELLITUS}

\section{ABSTRACT}

Acute complications of diabetes (hypoglycemia, diabetic ketoacidosis, hyperosmolar hyperglycemic state and lactoacidosis) are life threatning conditions, related in most cases to acute illnesses and therapeutic failures. These complications can be prevented by patient education. The Family Physician must be prepared to diagnose and treat them and to refer the patient immediately to a specialized unit, especially when the patient is vomiting and showing signs of dehydration. 